\title{
Origin and control of ionic hydration patterns in nanopores
}

\author{
Miraslau L. Barabash ${ }^{1, \#}$, William A. T. Gibby ${ }^{1}$, Carlo Guardiani ${ }^{1, *}$, \\ Alex Smolyanitsky ${ }^{2}$, Dmitry G. Luchinsky ${ }^{1, \S}$, Peter V. E. McClintock ${ }^{1}$ \\ ${ }^{1}$ Department of Physics, Lancaster University, Lancaster, UK, LA1 4 YB \\ ${ }^{2}$ Applied Chemicals and Materials Division, National Institute of Standards and Technology, Boulder, CO 80305, USA \\ ${ }^{*}$ Present address: Department of Mechanical and Aerospace Engineering, Sapienza University, 00185 Rome, Italy \\ ${ }^{\S}$ Present address: KBR Inc., Ames Research Center, Moffett Field, CA, USA
}

(Dated: April 30, 2021)

\begin{abstract}
In order to permeate a nanopore, an ion must overcome a dehydration energy barrier caused by the redistribution of surrounding water molecules. The redistribution is inhomogeneous, anisotropic and strongly position-dependent, resulting in complex patterns that are routinely observed in molecular dynamics simulations. Here, we study the physical origin of these patterns and of how they can be predicted and controlled. We introduce an analytic model able to predict the patterns in a graphene nanopore in terms of experimentally accessible radial distribution functions, giving results that agree well with molecular dynamics simulations. The patterns are attributable to a complex interplay of ionic hydration shells with water layers adjacent to the graphene membrane and with the hydration cloud of the nanopore rim atoms, and we discuss ways of controlling them. Our findings pave the way to designing required transport properties into nanoionic devices by optimising the structure of the hydration patterns.
\end{abstract}

Keywords: artificial nanopore, control, design, ionic hydration shells, radial distribution function (RDF)

\section{Introduction}

Ionic permeation through narrow water-filled channels and pores is of profound importance in both biophysics and technology [1, 2, finding applications in fuel cells 3], biological ion channels [4, water desalination [5], gas 9] and isotope [10] separation, DNA sequencing [11, 12], and "blue energy" harvesting [12 14. Ångström-sized pores in artificially fabricated membranes are promising candidates for reproducible ondemand engineering and control of selective ionic and molecular transport.

It is well-known that the structure of ionic hydration shells is one of the key factors controlling ionic permeation in nanopores [8, 15 17. In confined geometries some of the water molecules are lost from the hydration shells, and the resultant dehydration barrier is the subject of extensive research [8, 17,28$]$. One outcome is an appreciation of the extraordinary complexity of the hydration patterns around an ion in the pore [18, 22, 29], as has been confirmed by numerous molecular dynamics (MD) simulations [5, 8, 22, 30, 31]. The latter reveal that the patterns are highly inhomogeneous, anisotropic and spatially clustered near membranes 32, inside nanotubes 33, and in nanoslits [16, 34. This clustering determines the distribution of charge around the ion, the local dielectric permittivity 35, 36, and the strength of

\footnotetext{
\# Electronic addresses: miroslav.barabash@gmail.com (MLB, corresponding author), w.gibby@lancaster.ac.uk (WATG), carlo.guardiani@uniroma1.it alex.smolyanitsky@nist.gov (AS),dmitry.g.luchinsky@nasa.gov (DGL), p.v.e.mcclintock@lancaster.ac.uk (PVEM)
}

the ions' electrostatic interactions with their surroundings [37. However, to understand, characterise, and predict the hydration patterns theoretically is a challenging problem [2] that hitherto has remained unresolved.

We now develop an analytic model that can predict the hydration patterns of ions in nano-confinement through use of radial distribution functions (RDFs), evaluated in the bulk electrolyte. We show that the patterns result from interference between the ionic hydration shells, water layering near the membranes and the hydration cloud around the nanopore. We compare the analytically predicted patterns with the results of MD simulations for different ionic locations near graphene nanopores with a diversity of shapes, geometries and strains. The results are applicable to narrow biological channels [24, 38, 39, functionalized sub-nanopores [24, 25, 40, 41], nanotubes [42] and nanoslits [16, 34. Thus, this work takes an important step towards controlling the function of nanoscale devices by tailoring the hydration patterns.

\section{Results}

Model. We consider graphene lattice 43 with nanopore and an ion (e.g. $\mathrm{K}^{+}$) in a water box, see Fig. 1. The pore is created by removing one hexagon of carbon atoms from near the geometrical centre of the lattice.

For simplicity, we consider the carbons and the ion as being clamped. The spatial distribution of water oxygens is given by

$$
\begin{aligned}
& \rho\left(\mathbf{r}_{1}^{\mathrm{w}} \mid\left\{\mathbf{r}_{m}^{\mathrm{c}}\right\}, \mathbf{r}^{\mathrm{i}}\right)= \\
& \frac{1}{Z} \int \ldots \int \mathrm{e}^{-\beta H\left(\mathbf{r}_{1}^{\mathrm{w}}, \ldots, \mathbf{r}_{N}^{\mathrm{w}}, \mathbf{p}_{1}^{\mathrm{w}}, \ldots, \mathbf{p}_{N}^{\mathrm{w}} \mid\left\{\mathbf{r}_{m}^{\mathrm{c}}\right\}, \mathbf{r}^{\mathrm{i}}\right)} \\
& \times \mathrm{d} \mathbf{r}_{2}^{\mathrm{w}} \ldots \mathrm{d} \mathbf{r}_{N^{\mathrm{w}}}^{\mathrm{w}} \mathrm{d} \mathbf{p}_{1}^{\mathrm{w}} \ldots \mathrm{d} \mathbf{p}_{N^{\mathrm{w}}}^{\mathrm{w}}
\end{aligned}
$$




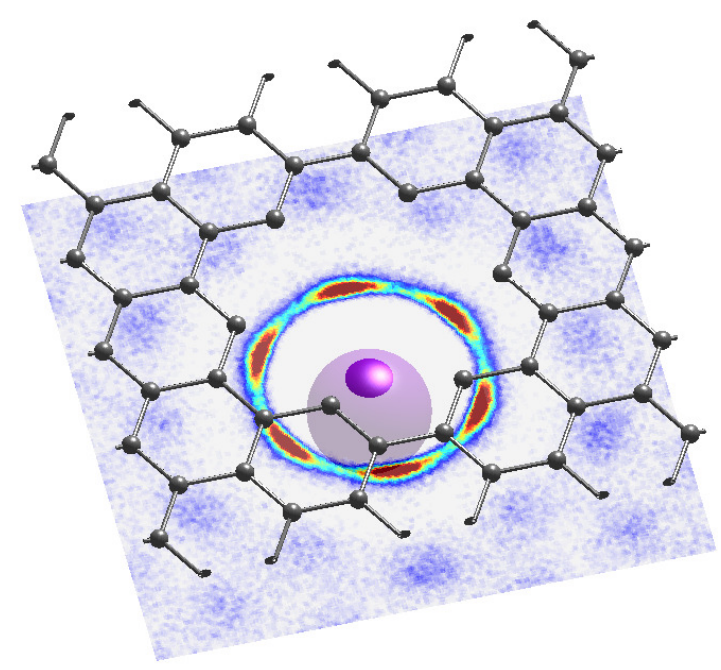

FIG. 1. Model setup. A hexagonal pore in pristine graphene (gray balls and sticks) and a $\mathrm{K}^{+}$ion (purple sphere) centered at $Z=-0.4 \mathrm{~nm}$. The color plane represents the density pattern of water oxygen atoms in the plane at $Z=-0.28 \mathrm{~nm}$. (There will be comparable colour planes at other $Z$ values.) The colours indicate density values relative to the bulk, brown being the highest (4) and dark blue being the lowest (0) value. The density has been evaluated by MD simulation, and it is compared with theory, Eq. (4), in Supplementary Figure 1.

where $H(\cdot)$ is the all-atom Hamiltonian, comprising the water-ion $U_{\mathrm{iw}}$, water-lattice $U_{\mathrm{ic}}$ and water-water $U_{\mathrm{ww}}$ interactions; $Z$ is the partition function, $\beta=1 / k_{B} T, k_{B}$ is Boltzmann's constant and $T$ is the absolute temperature. Integration over momenta $\mathbf{p}_{n}^{\mathrm{w}}$ leads to a factor $\left(2 \pi m^{\mathrm{w}} k_{B} T\right)^{3 N^{\mathrm{w}} / 2}$ which subsequently cancels out due to normalization. Here $\left\{\mathbf{r}_{n}^{\mathrm{w}}\right\},\left\{\mathbf{r}_{m}^{\mathrm{c}}\right\}, \mathbf{r}^{i}$ represent the 3D coordinates of the water molecules, atoms of the lattice, and the ion, respectively. Water molecules can be chosen arbitrarily, and so we omit the sub-index in $\mathbf{r}_{1}$ for clarity, so that $\mathbf{r}$ is used hereafter. Supplementary Discussions 1 and 2 in Supplementary Methods provide a more detailed derivation of the quantities in this section.

Direct application of the analytic formula (1) is not feasible due to the large number of degrees of freedom in the solution. However, statistical averaging over all water molecules generates rigorously the potential of the mean force (PMF) 44 46] which can be approximated as [47, p.77]

$$
W\left(\mathbf{r} \mid\left\{\mathbf{r}_{m}^{\mathrm{c}}\right\}, \mathbf{r}^{\mathbf{i}}\right)=\mathcal{W}_{\mathrm{iw}}\left(\mathbf{r}-\mathbf{r}^{\mathbf{i}}\right)+\sum_{m}^{N^{\mathrm{c}}} \mathcal{W}_{\mathrm{cw}}\left(\mathbf{r}-\mathbf{r}_{\mathbf{m}}^{\mathbf{c}}\right) .
$$

Note that the interactions of the ion or carbon atoms with other water molecules are already incorporated implicitly within the PMF. Note also that a similar strategy - construction of a multi-ion PMF from sphericallysymmetrical pairwise components measured in separate MD simulations - has been used successfully in an atomic-resolution Brownian dynamics study of the ionic selectivity of $\alpha$-Hemolysin [48] and to describe DNA translocation through nanopores [49].

Importantly, the water PMF is related rigorously to the correlation function $\mathcal{W}(\mathrm{CF})$ via [44,46]

$$
\mathcal{W}(\mathbf{r})=-\beta^{-1} \ln g(\mathbf{r}),
$$

assuming that $\mathcal{W} \rightarrow 0$ and $g(\mathbf{r}) \rightarrow 1$ as $\mathbf{r} \rightarrow \infty$. We evaluate the RDFs in bulk through separate MD simulations (see Discussions 1 and 2 of Supplementary Methods for details). Note that this needs to be done only once for a given density $\rho_{0}$ and temperature $T$ [50.

The relations (2) and (3) allow one to rewrite the water spatial density $\rho(\mathbf{r})$ in the form

$$
\frac{\rho\left(\mathbf{r} \mid\left\{\mathbf{r}_{m}^{\mathrm{c}}\right\}, \mathbf{r}^{\mathbf{i}}\right)}{\rho_{0}}=g_{\mathrm{iw}}\left(\left|\mathbf{r}-\mathbf{r}^{\mathbf{i}}\right|\right) \cdot \prod_{m}^{N^{\mathrm{c}}} g_{\mathrm{cw}}\left(\left|\mathbf{r}-\mathbf{r}_{\mathbf{m}}^{\mathbf{c}}\right|\right) .
$$

where $\rho_{0}$ is the bulk water density (see Supplementary Discussion 1 for details). Note that the modulus sign reduces the 3D ion-water and carbon-water interactions to ion-oxygen and carbon-oxygen interactions, respectively. Thus, one is relying on the radial density functions (RDFs) $g(r)$ which are one-dimensional functions of distance, in contrast to three-dimensional CFs.

In the derivation we take no explicit account of waterwater interactions, in particular between their oxygen atoms. This simplification leads to the water being represented as an ideal gas, and it overestimates the water density near the graphene walls. This can clearly be seen near the graphene lattice in the lower panels of Fig. 2.

Equation (4) allows one to compute the density of the water wetting a given set of atoms as a product of component RDFs, and to evaluate the water PMF via the Boltzmann inversion (3). Equation (4) also shows that the water pattern results from interference between the ionic hydration shells ( $g_{\text {iw }}$ term) and the water layers surrounding the graphene membrane ( $\prod g_{\mathrm{cw}}$ term). Supplementary Discussion 3 illustrates the emergence of the hydration pattern when groups of carbon atoms are added sequentially to compose a nanopore. Equation (4) represents the well-known Kirkwood superposition approximation [50, 51, [52, p.186]. It has also been generalized to describe the ice-water interface [50] and solvated DNA [53. Here, we apply this relation for the first time to describe the water density patterns around an ion near an artificial nanopore.

The usefulness of an approach based on Eq. (4) is twofold. First, use of the bulk RDF values to describe water density near the pore, provides for a $10^{2}-10^{4}$ speedup in comparison with all-atom MD simulations [50]. In turn, the single-component bulk RDFs $g(r)$ can be obtained in a number of ways. The most straightforward is to use experimental values derived from the structure factor measured by X-ray [54] or neutron [55] diffraction. Thus one can avoid uncertainties related to the properties of the force field (parametrization, polarizability) 

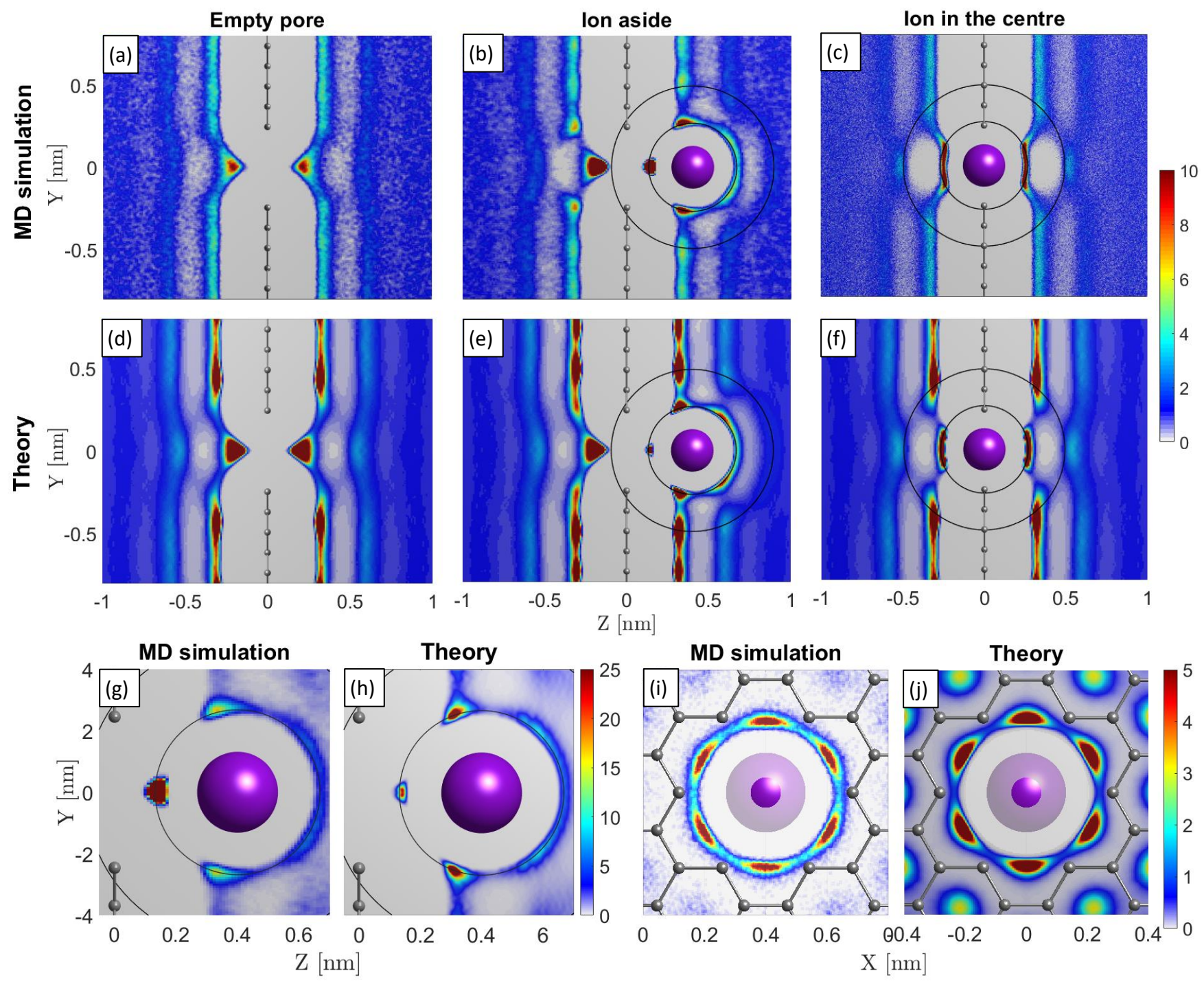

MD simulation

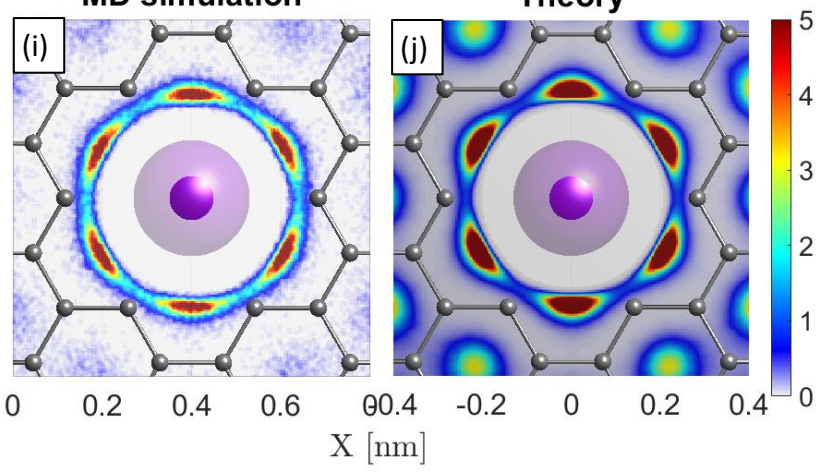

FIG. 2. Comparison of analytic theory with MD simulations. The graphene sheet is shown in section by the vertical line of carbon atoms, in which the pore appears as a centrally-placed gap with horizontal axis of symmetry. The $\mathrm{O}^{-}$density in the vicinity of an ion (purple sphere) near the pore, derived from the MD simulations (a)-(c) is compared with the predictions of Eq. (4) (d)-(f). Panels (a) and (d) show the oxygen density around an empty pore; (c) and (f) show the density for a $\mathrm{K}^{+}$ ion in the centre of the pore; (b) and (e) show the density when the ion is centred $0.4 \mathrm{~nm}$ from the entrance to the pore. The fine structure of the oxygen density pattern around a $\mathrm{K}^{+}$ion at the pore centre, obtained from MD simulations (g,i) and from theory Eq. (4) $(\mathrm{h}, \mathrm{j})$. The concentric black circles represent the first two maxima of the $\mathrm{K}^{+}-\mathrm{O}^{-} \mathrm{RDF}$, and are given as guides to the eye. The graphene lattice is indicated in a grey ball-and-stick representation.

and thus connect the experimentally-measurable structural properties of the bulk electrolyte solution with those near a nanopore. Other approaches rely on MD, MonteCarlo or DFT simulations, or on the integral theory of liquids [46, 56 59]. In the present work, MD-derived RDFs have been chosen in order to simplify the comparison between the MD simulations and analytics.

Secondly, the method can be readily extended to any material or pore geometry. The latter paves the way towards changing the pore parameters - size, shape, number of layers, strain tensor, rim charge, functional groups) and choice of lattice type (e.g. $\mathrm{MoS}_{2}, \mathrm{hBN}, \mathrm{WS}_{2}$, etc. as well as graphene) - with the purpose of changing the dehydration pattern. The pattern defines the dehydration energy profile and thus determines the pore's permeability and selectivity, which are vital for inverse-designing the function of Ångström-scale ionic devices. In Supplementary Discussion 4, we consider a tentative analytical way of connecting the hydration patterns and the singleion PMF. Below we compare the ionic hydration patterns, obtained from MD simulations, with those from theory (Eq. (4)). 
(a)

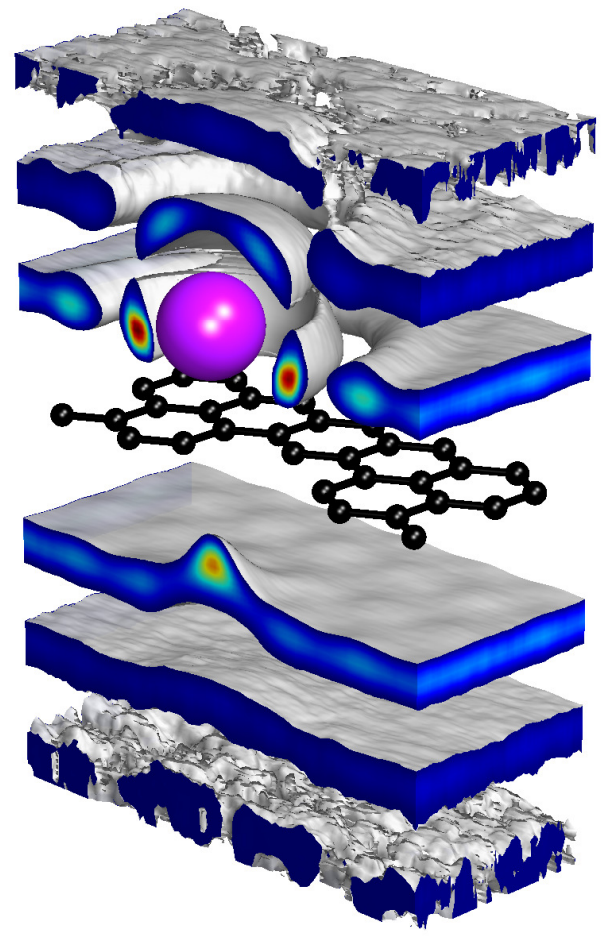

(b)

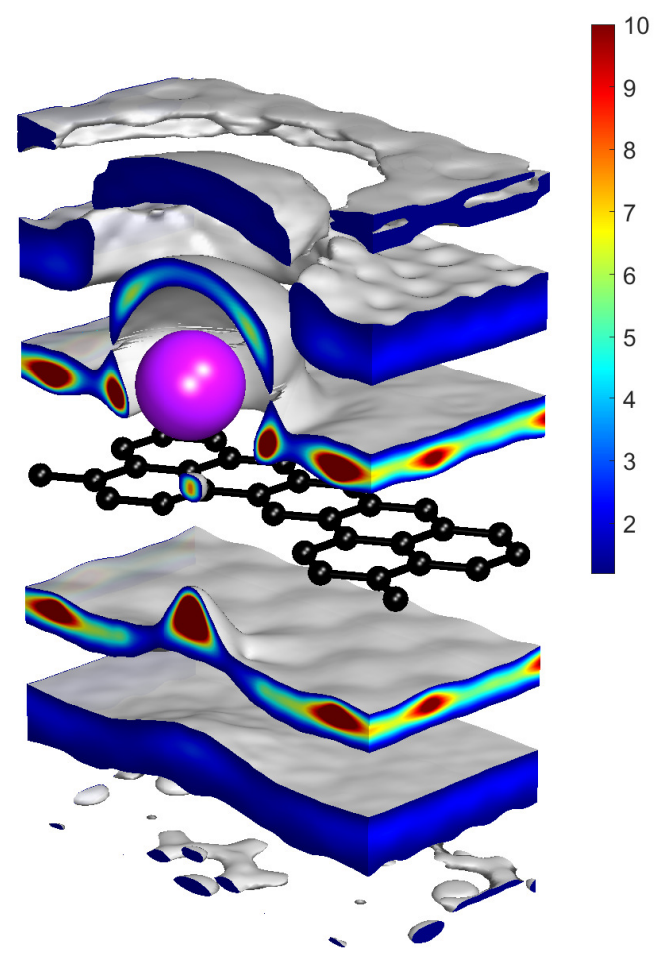

FIG. 3. Three-dimensional ionic hydration patterns. Water distribution around a $\mathrm{K}^{+}$ion (purple sphere) at $Z=0.4 \mathrm{~nm}$ near the pore in a graphene lattice (black ball-and-stick representation), obtained from (a) MD simulations and (b) theory, Eq. (4). An isovalue of 1.15 was chosen to reveal the layered structure of the hydration around the ion and the graphene lattice. Both panels have been smoothed with a 5-point window.

Molecular dynamics simulation results. Figure 2 compares the MD results (top row) with the corresponding theoretical predictions, Eq. (4) (middle row). They agree well in that the qualitative features of the water distributions are well reproduced: as described by the $\prod g_{\text {iw }}$ term in Eq. (4), superposition of the contributions from all the carbon atoms results in a decaying plane density wave in the $z$-direction, matching the troughs and peaks of the C-OH RDFs: see Supplementary Figure 2. The presence of the pore in an otherwise intact lattice distorts the water layers near the membrane, and allows water molecules to exist in the pore as revealed by the strength of their distribution.

An ion in the pore, Fig. 2(c), suggests sphericallysymmetric spatial density waves, as implied by the term $g_{\text {iw }}$ in Eq. (4). The resultant superposition of the plane wave from the graphene with the spherically-symmetrical hydration shells from the ion leads to a reshaping of the density such that the first hydration shell, while partially remaining, becomes significantly altered. In particular, it acquires maxima where the $\mathrm{O}-\mathrm{C}$ and $\mathrm{O}-\mathrm{K}^{+} \mathrm{RDF}$ maxima cooincide, minima when both RDFs reach minima, and mid-values where the maximum of one RDF overlaps with the minimum of another. Circular waves in
Fig. 2 (b,c,e,f) around the ion correspond to the first two maxima of the intact bulk $\mathrm{O}-\mathrm{K}^{+} \mathrm{RDF}$, indicated by the concentric circles at their respective radii. The density around the centrally-located ion preserves the first hydration shell, but with a modulation due to the graphene atoms; the structure of the second shell is significantly affected by interactions with carbon atoms. Thus, the observed water patterns arise from interference between the ionic hydration shell and the hydration cloud around the nanopore. Analysis of the hydration patterns around an $\mathrm{Na}^{+}$and $\mathrm{Cl}^{-}$ions, shown in Supplementary Note 1, reveals similar features.

The central parts of Fig. 2(b) and (e) are shown at higher resolution in panels $(\mathrm{g})$ and $(\mathrm{h})$. One can see the fine structure in the water density pattern resulting from the superposition of the ion-oxygen and carbon-oxygen interactions. The density crescent at $Z \approx-0.25 \mathrm{~nm}$ in Fig. 2 (g) and (h) is induced by the first maximum of the RDF, as indicated by the concentric circles, while the two peaks appear due to the oxygen interaction with the carbon atoms. The low-density area around $[-4,0]$ emerges due to the minimum of the $\mathrm{K}^{+}-\mathrm{O}^{-} \mathrm{RDF}$ located at around $0.35 \mathrm{~nm}$. The "island" around $[0.175,0] \mathrm{nm}$ indicates the position of the trapped water molecule, and 

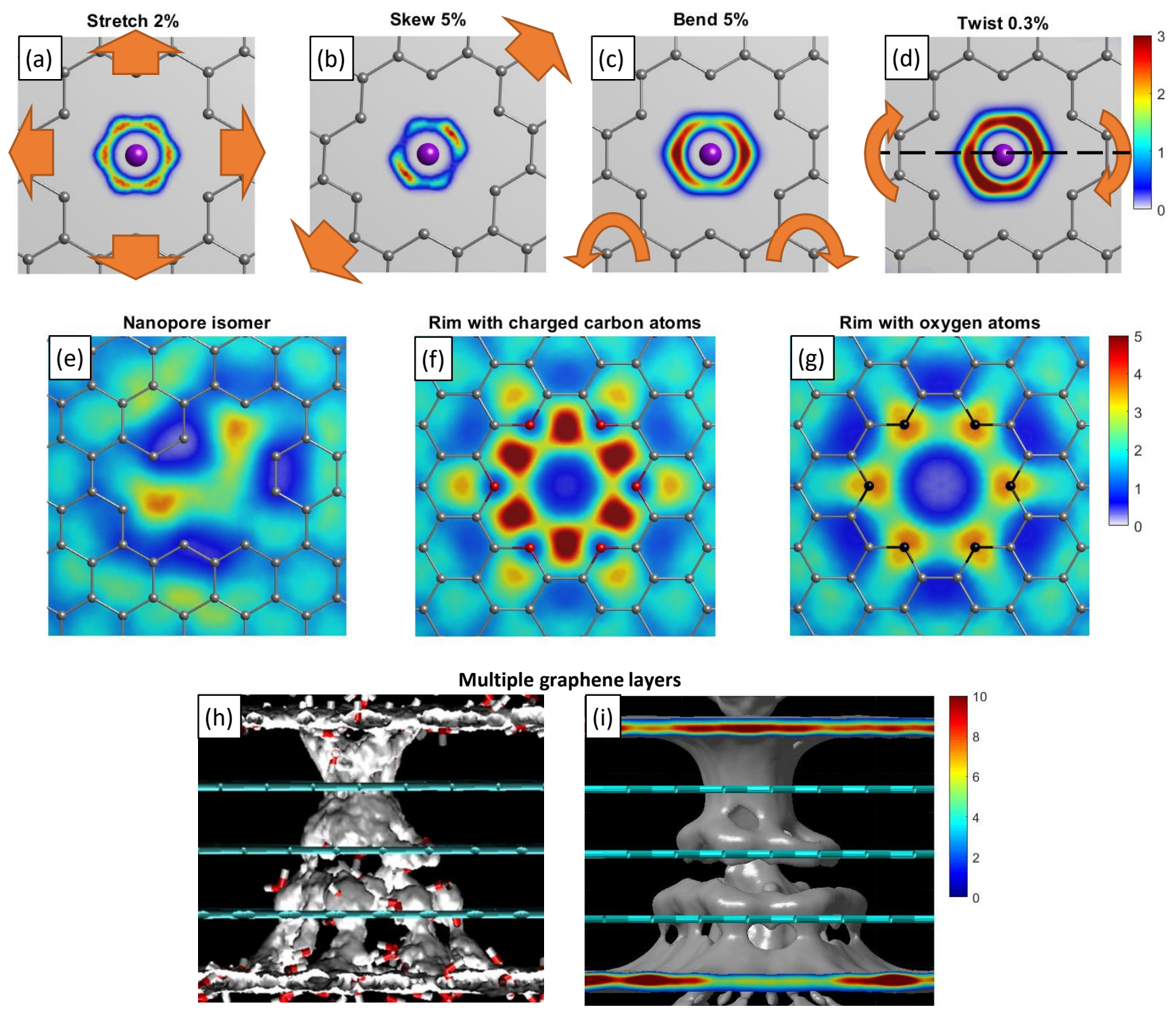

FIG. 4. Tools to control the hydration pattern. These include the extrinsic processes of: (a) stretching, (b) skewing or asymmetric stretching, (c) bending, (d) twisting, and the intrinsic features of - (e) choice of pore isomer, (f) charging of the rim atoms (red balls), (g) functionalising the rim with oxygen atoms (black balls), (h) use of a multi-layer pore, as exemplified in a molecular dynamics simulation (Fig. 4(h) adapted from ref. 60, copyright 2019, with permission from Elsevier), and (i) theoretical prediction, Eq. (4). Density slices have been taken at $Z=-0.23 \mathrm{~nm}$ (a-d) and $Z=-0.28 \mathrm{~nm}$ (e-g). The graphene lattice is indicated in a grey ball-and-stick representation.

highlights the predictive power of the proposed analytical method (see Supplementary Note 2 for more details). The cross-sections of the crescents in Fig. 2 (g) and $(\mathrm{h})$, made at $0.25 \mathrm{~nm}$, are shown in panels (i) and (j). These arise from an interplay between the oxygen-ion and oxygen-pore interactions: the minimum at the origin arises due to the convex shape of the first hydration shell near the ion, while the hexagonal structure is inherited from the hexagonal geometry of the graphene pore (gray balls and sticks). Similar water density patterns in the plane of the nanopores have been observed in numerous

\section{MD simulation works $[8,17,18,22,30$ recently.}

Figure 3 combines the previous results by illustrating the $3 \mathrm{D}$ isosurfaces of the hydration shells around the ion and the pore, taken at a level of 1.15. Panels (a) and (b) show that the results from MD and theoretical prediction Eq. (4), respectively, agree well. These figures reproduce the layering of water near the graphene sheet, with first three layers visible (see Supplementary Note 3 for details), as well as the spherical hydration shells around the $\mathrm{K}^{+}$ion. Parts of the preserved $1^{\text {st }}$ and $2^{\text {nd }}$ hydration shells are clearly evident. The figure emphasises 


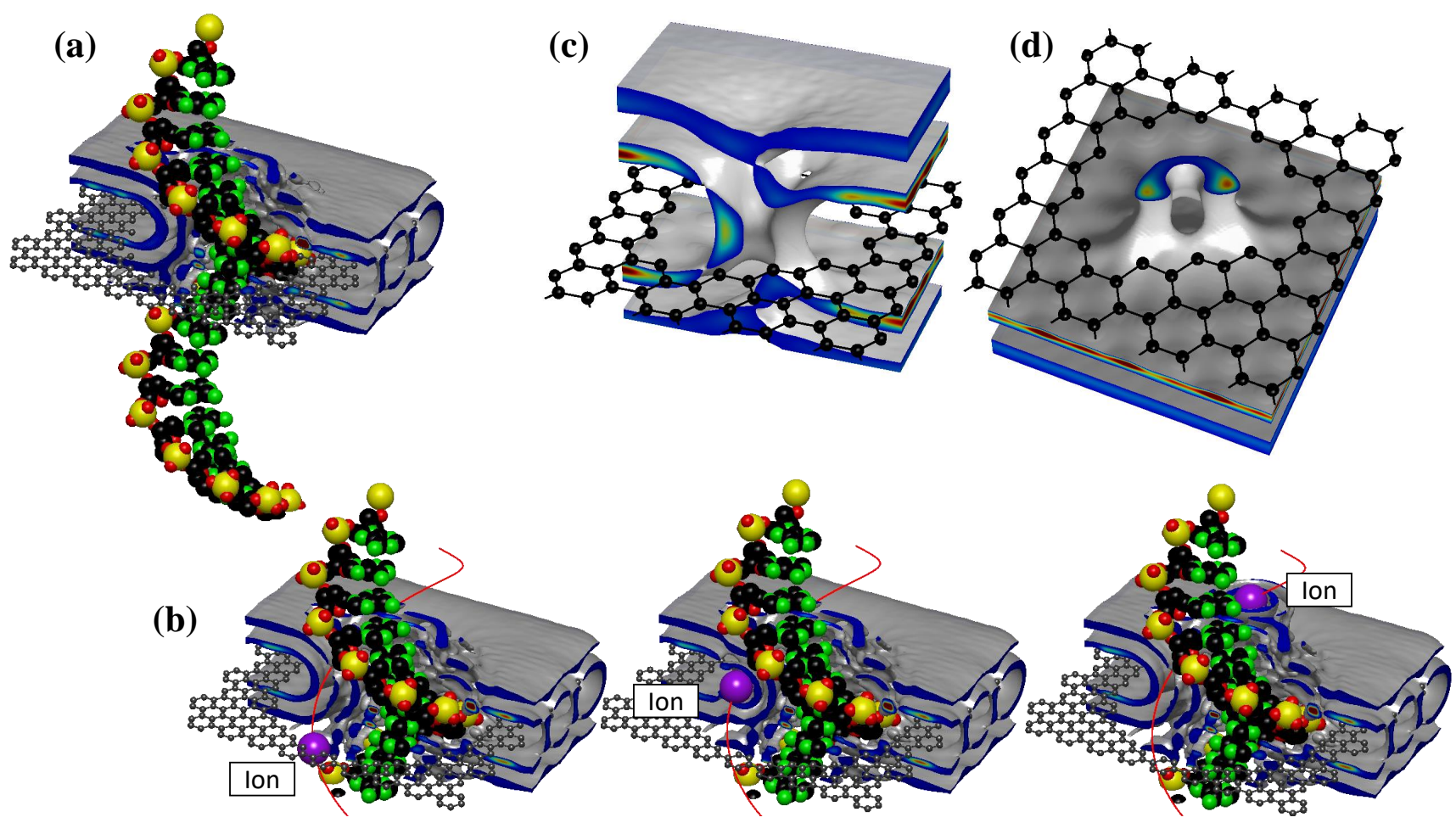

FIG. 5. Possible applications of our proposed method to DNA sequencing and the cataloguing of the solvated nanopore isomers. (a) Hydration pattern wetting the single-stranded DNA in a nanopore. Densities are obtained from Eq. (4) and are shown as grey isosurfaces (isovalue=1.2) and colored cross-sections. The DNA is represented by connected colored spheres (oxygen (red), nitrogen (green), carbon (black), phosphorus (yellow)). Graphene lattice is shown in grey balls and sticks. (b) Three consecutive snapshots of the $\mathrm{K}^{+}$ionic trajectory along the groove of the DNA, depicted by the red line. (c) and (d) represent the nontrivial structure of the solvation patterns around one of the pore isomers, taken from Ref. 61] (isomer for the case of $N=19$, the frequency of observation of the isomer - 4\% -, total number of possible isomers - 488 [61]). The isosurfaces are calculated at the isovalue of 1.2. Graphene lattice is shown in black balls and sticks.

the inhomogeneity and anisotropy of the water patterns emerging from the combined effects of the ion and the graphene lattice. The origin of multiple water layers is illustrated in Supplementary Figure 12.

We have also studied how the hydration structure evolves as the ion's position changes. The results are summarised in Supplementary Note 4 and the two Supplementary frame series (Supplementary Movie 1 and Supplementary Movie 2). These sets clearly illustrate that the water structure is significantly modified, even when the ion is located more than $1 \mathrm{~nm}$ away from the nanopore. In other words, when the lattice- (hydration layers) and ion-induced (hydration shells) inhomogeneities begin to overlap, the ion-pore interaction starts to change. This result supports the notion that solventmediated interactions extend the range of the interactions with the pore by a large factor compared with the bare ion radius [62].

Finally, in Fig. 4 we illustrate the predicted effects of the set of tools that we propose for controlling the dehydration pattern. They can be categorized into two groups. The first set is extrinsic and includes (a) stretching, (b) skewing or asymmetrical stretching, (c) bend- ing, and (d) twisting of the lattice (see Supplementary Notes 8-11). Secondly, we propose adjustments of some intrinsic features: (e) the nanopore geometry, (f) rim charge, (g) atom type and (i) layer number (see Supplementary Notes 12-17). Our theory yields good agreement with the published crown-like water density patterns (panel (h)) from Ref. 61. More examples of these tools in action are given in Section 3 of the Supplementary Information.

It is evident that Eq. (4) can reproduce well the qualitative features of the water distribution near the pore given in Figs. 2. We point out, however, that there is a numerical discrepancy - a factor of $\sim 3$ - between the peak values of the maps, clearly seen between the MDgenerated and theoretical density of the "ridges" near the graphene lattice. We attribute this discrepancy to our omission of the water-water interactions in Eq. (4), which would imply an absence of short-range repulsion between oxygen or hydrogen atoms of separate water molecules, so that arbitrarily small separations can be realised. The consequence is a denser water distribution, localized near the carbon atoms. Also, the reduction from a full 3D model to the radial CF picture overlooks the water-water 

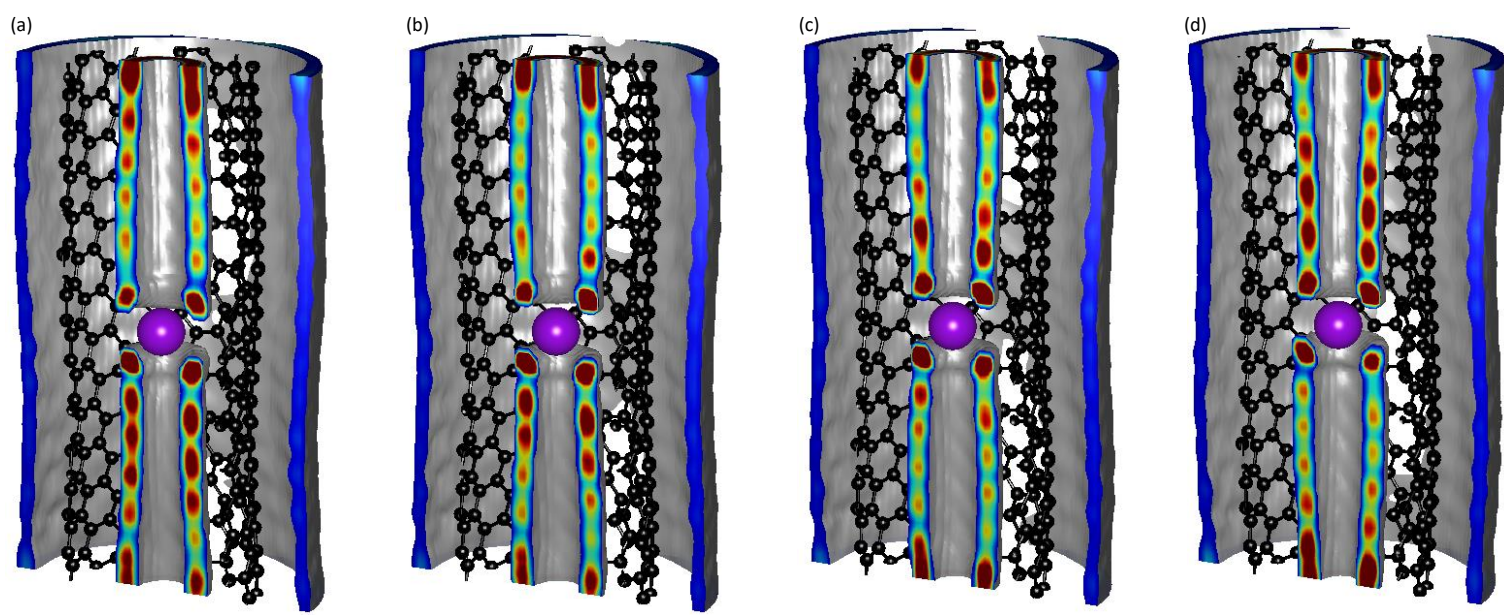

FIG. 6. Water density patterns in a $(\mathbf{5 , 1 0 )}$ CNT with a mechanical wave travelling along it. Panels (a)-(d) show frames taken at intervals $t_{m}=\left(\frac{0}{6}, \frac{1}{6}, \frac{2}{6}, \frac{3}{6}\right) T$, where $T$ is the period of the wave. This provides an illustration of how a transverse wave, traveling along the CNT axis, may lead to the directed motion of ions against the electrochemical gradient, thus providing a potential design for mechanically-induced ion-pumping. See Supplementary Movie 5 for a visualization.

relative orientations and thus limits the applicability of the method in carbon nanotubes. We are currently working on eliminating these shortcomings of the technique by using higher-dimensional CFs [50, and applying machine learning (see Supplementary Discussion 5 for a toy example).

\section{Discussion}

Our approach can readily be extended to account for the density of hydrogen atoms, and thus to evaluate the average charge density in the domain. Doing so promises deep insight into water-mediated interactions, including the pore rim's hydration [63] and reorientation of the water dipoles in confinement which reduces [64, 65] the dielectric permittivity. The latter is needed for quantifying the ion and polymer interactions with the pore and with the external electric field and for understanding the ionic diffusivity [66] in biological channels, as well as the properties of the electric double layer [67, 68. The method can also be used to define the demarcation interface between the pore and the protein in continuous theories of ion transport such as the Poisson-Nernst-Planck (PNP) theory [69. The method can thus provide fundamental insights into the electrostatics and dynamics of ions in sub-nanopores, where the dielectric properties on the nanoscale remain a challenging unsolved problem that is subject to intensive research [35, 36, 70, 71].

Our results demonstrate the applicability of the method to describing water density patterns under quite general conditions, e.g. for arbitrary ionic positions, pore geometry (size, shape, number of layers, offset eclipse [72]), stretching, skewing, bending, and twisting of the lattice, the type of atom around the rim of the pore, and the nature of the 2D material 73 . This opens the way to multi-parameter optimization 74 of the energy landscape by adjustment of the corresponding hydration patterns. For example, by iteratively choosing the most appropriate combination of pore geometric parameters, and subsequently manipulating the pore shape externally (e.g. by stretching), the nanopore's permeability and selectivity can be optimised and regulated, providing for permeation along specified pathways [75] 77 . This approach could prove useful in addressing two highly topical technological challenges: first, water desalination, to maximise the quality and energy efficiency while minimizing the environmental impact of the process [7); and secondly, energy harvesting, to maximize energy conversion by minimising Joule heating, thereby raising the output power density above the level of $5 \mathrm{~W} / \mathrm{m}^{2}$ needed to make harvesting economically viable [12, 14].

The same design and optimisation strategy should serve to slow down the translocation and guide the orientation of DNA (see Fig. 5(a) for a possible example), which constitutes one of the current challenges to fast genome sequencing [11, 78]. Likewise, it is believed that the accuracy of nucleobase detection using the Coulter principle can benefit from an optimisation of the features of the nanopore, Fig. 5(b) (see also Supplementary Movies 3, 4, and Note 5). Our approach would allow one to catalogue solvated $2 \mathrm{D}$ and $3 \mathrm{D}$ pore isomers 61, as illustrated in Fig. 5(c) and (d), thus benefiting the Materials Genome Initiative [79, 80. Finally, by applying a travelling transverse (corrugated) wave one might be able to create a nanoscale ionic pump driving ions against the electrochemical gradient [81] (see Fig. 6. Supplementary Movie 5 and Note 6). Thus, the proposed method paves the way to the optimisation and design of novel nanoionic devices in many contexts and applications 82 .

\section{Conclusions}

We have proposed and validated an analytic method for revealing and quantifying the hydration patterns around 
an ion near a sub-nanopore, using a product of the radial density functions (RDFs) measured in the bulk electrolyte. The complexity and fragmentation of these patterns result from interference between ionic hydration shells and the hydration cloud around the nanopore. The method can be extended to account for the distribution of hydrogen atoms, and hence to obtain insight into local electrostatic interactions on the nanoscale. It is fast, and it allows one to take explicit account of the nanopore's detailed geometry (size, length, shape, number of layers, offset eclipse), pore constituents (charge and atom type), and of the ion's position, as well as implicitly to take account of the ion type, solvent, temperature, and pressure. Multi-parameter optimization of the pore parameters and surroundings is expected to create a way of engineering and regulating the energy barrier in nanopores via control of the hydration pattern. This strategy opens a new avenue to designing, optimising, and controlling the permeability and selectivity properties of nanoionic devices, as well as regulating the translocation of DNA through solid-state nanopore sequencers.

\section{Methods}

\section{Molecular dynamics simulation details}

We used VMD 83 to build the systems in question, and NAMD2 84, with the CHARMM27 force field for molecular dynamics simulations at $T=300 \mathrm{~K}$ with a timestep of $1 \mathrm{fs}$ and the velocity Verlet algorithm. The Lorentz-Berthelot combining rule was applied. The TIP3P 85] water model was used. A cutoff of $1.2 \mathrm{~nm}$ for non-bonded interactions (Lennard-Jones and Coulomb) with a switching distance of $10.0 \AA$ (1nm) was used, and the full electrostatic calculation was performed using the Particle Mesh Ewald (PME) scheme [86]. Periodic boundary conditions were imposed in all directions.

All lattice atoms were fixed by setting the beta parameter to 1 . The system first underwent equilibration during the initial 1000 steps in the Nosé-Hoover thermostat at pressure $p=1$ atm $(101.325 \mathrm{kPa})$, with the remaining simulation running under NVT conditions. The frames were captured every 100 steps. No external electric field was applied. Unless otherwise stated, typical production runs took $10 \mathrm{~ns}$ and were sampled every 100 steps to yield statistically significant figures.

RDFs were measured for a free atom $\left(\mathrm{C}, \mathrm{K}^{+}, \mathrm{Na}^{+}\right)$in the water surroundings. When an ion's RDF was measured, the corresponding number of free counterions $\left(\mathrm{Cl}^{-}\right.$ for $\mathrm{K}^{+}$, and $\mathrm{K}^{+}$for $\mathrm{Cl}^{-}$) was added to neutralize the system. Finally, RDFs were measured using the VMD plugin gofr. Molecular structures were visualized and rendered using VMD 83 .

\section{Data analysis}

MD trajectories were read by an readdcd.m and readpdb.m scripts MDToolbox [87] for MATLAB [88]. A home-made script to build and analyse the histrogram distributions included built-in MATLAB functions and some external functions: histcn.m [89], smooth2a.m [90]. All densities were normalised by the bulk values. A $3 \times 3$ - point smoothing window was applied to suppress the pixelation of the MD histograms, unless otherwise stated.

\section{Acknowledgements}

We are grateful to N. D. Drummond, B. Luan, A. Stefanovska and N. G. Stocks for valuable discussions. The work was partially supported by a PhD Scholarship from the Faculty of Science and Technology of Lancaster University, by the Engineering and Physical Sciences Research Council UK (grants EP/M016889/1 and EP/M015831/1), and by a Leverhulme Trust Research Project Grant RPG-2017-134. CG is currently supported by a project that has received funding from the European Research Council (ERC) under the European Union's Horizon 2020 research and innovation programme (grant agreement No. 803213). MLB, CG, WATG, DGL and PVEMcC gratefully acknowledge the use of the High End Computing facility at Lancaster University. AS gratefully acknowledges support from the Materials Genome Initiative.

Certain commercial equipment, instruments, or materials are identified in this paper in order to specify the experimental procedure adequately. Such identification is not intended to imply recommendation or endorsement by NIST, nor is it intended to imply that the materials or equipment identified are necessarily the best available for the purpose.

\section{Author contributions}

M.B., A.S. and D.L. conceived the central ideas. M.B. developed the theory, devised and implemented the analysis software. M.B., D.L., W.G. and at the early stage C.G. designed and performed MD simulations. P.V.E.McC. oversaw the project, discussed the results, and helped with the manuscript. All authors discussed the results, contributed to the writing and editing of the manuscript, and approved the final version.

\section{Competing interests}

The authors declare no competing interests.

\section{Additional information}

Supplementary Information is available for this paper.

\section{Code availability}

MATLAB codes for computing the ionic hydration patterns according to Eq. (4) and creating a movie analogous to Supplementary Movie 2, and transforming the crystal lattice are available as ZIP archives in Supplementary Data 1 and 2 (see Supplementary Note 7 for details). Other codes that support the results reported in this paper and other findings of this study are available from the corresponding author upon reasonable request.

\section{Data availability}

The datasets generated during and/or analysed during the current study are available from the corresponding author on reasonable request.

\section{References}

[1] Bond, A. H., Dietz, M. L. \& Chiarizia, R., Incorporating size selectivity into synergistic solvent extraction: A 
review of crown ether-containing systems, Industrial $\&$ Engineering Chemistry Research 39, 3442-3464 (2000).

[2] Faucher, S. et al., Critical knowledge gaps in mass transport through single-digit nanopores: A review and perspective, J. Phys. Chem. C 123, 21309-21326 (2019).

[3] Wang, Y., Chen, K. S., Mishler, J., Cho, S. C. \& Adroher, X. C., A review of polymer electrolyte membrane fuel cells: Technology, applications, and needs on fundamental research, Appl. Energy 88, 981 - 1007 (2011).

[4] Hille, B., Ion Channels of Excitable Membranes (Sinauer Associates Inc, 2001), $3^{\text {rd }}$ edn.

[5] Cohen-Tanugi, D. \& Grossman, J. C., Water desalination across nanoporous graphene, Nano Lett. 12, 3602-3608 (2012), pMID: 22668008.

[6] Rollings, R. C. \& Kuan, Aaron T. Golovchenko, J. A., Ion selectivity of graphene nanopores, Nat. Comm. 7 (2016).

[7] Lee, A., Elam, J. W. \& Darling, S. B., Membrane materials for water purification: design, development, and application, Environ. Sci.: Water Res. Technol. 2, 17-42 (2016).

[8] Fang, A., Kroenlein, K., Riccardi, D. \& Smolyanitsky, A., Highly mechanosensitive ion channels from grapheneembedded crown ethers, Nat. Mater. 18, 76-81 (2019).

[9] Du, H., Li, J., Zhang, J., Su, G., Li, X. \& Zhao, Y., Separation of hydrogen and nitrogen gases with porous graphene membrane, The Journal of Physical Chemistry $C$ 115, 23261-23266 (2011).

[10] Lozada-Hidalgo, M., Hu, S., Marshall, O., Mishchenko, A., Grigorenko, A. N., Dryfe, R. A. W., Radha, B., Grigorieva, I. V. \& Geim, A. K., Sieving hydrogen isotopes through two-dimensional crystals, Science 351, 68-70 (2016).

[11] Heerema, S. J. \& Dekker, C., Graphene nanodevices for DNA sequencing, Nat. Nanotechnol. 11, 127-136 (2016).

[12] Graf, M., Lihter, M., Altus, D., Marion, S. \& Radenovic, A., Transverse detection of DNA using a $\mathrm{MoS}_{2}$ nanopore, Nano Lett. 19, 9075-9083 (2019).

[13] Liu, G., Chen, T., Xu, J. \& Wang, K., Blue energy harvesting on nanostructured carbon materials, J. Mater. Chem. A 6, 18357-18377 (2018).

[14] Macha, M., Marion, S., Nandigana, V. V. R. \& Radenovic, A., 2D materials as an emerging platform for nanopore-based power generation, Nat. Rev. Mater. 4, 588-605 (2019).

[15] Joshi, R. K., Carbone, P., Wang, F. C., Kravets, V. G., $\mathrm{Su}$, Y., Grigorieva, I. V., Wu, H. A., Geim, A. K. \& Nair, R. R., Precise and ultrafast molecular sieving through graphene oxide membranes, Science 343, 752754 (2014).

[16] Esfandiar, A., Radha, B., Wang, F. C., Yang, Q., Hu, S., Garaj, S., Nair, R. R., Geim, A. K. \& Gopinadhan, K., Size effect in ion transport through angstrom-scale slits, Science 358, 511-513 (2017).

[17] Fang, A., Kroenlein, K. \& Smolyanitsky, A., Mechanosensitive ion permeation across subnanoporous $\mathrm{MoS}_{2}$ monolayers, J. Phys. Chem. C 123, 3588-3593 (2019).

[18] Zwolak, M., Lagerquist, J. \& Di Ventra, M., Quantized ionic conductance in nanopores, Phys. Rev. Lett. 103, 128102 (2009).

[19] Kaufman, I., Luchinsky, D. G., Tindjong, R., McClintock, P. V. E. \& Eisenberg, R. S., Energetics of discrete selectivity bands and mutation-induced transitions in the calcium-sodium ion channels family, Phys. Rev. E $\mathbf{8 8}$,
$052712(2013)$

[20] Kaufman, I. K., McClintock, P. V. E. \& Eisenberg, R. S., Coulomb blockade model of permeation and selectivity in biological ion channels, New J. Phys. 17, 083021 (2015).

[21] Kaufman, I. K. \& McClintock, P. V. E., Ionic Coulomb blockade, Nat. Mater. 15, 825-826 (2016).

[22] Sahu, S., Di Ventra, M. \& Zwolak, M., Dehydration as a universal mechanism for ion selectivity in graphene and other atomically thin pores, Nano Lett. 17, 4719-4724 (2017), pMID: 28678508.

[23] Guardiani, C., Fedorenko, O. A., Khovanov, I. A. \& Roberts, S. K., Different roles for aspartates and glutamates for cation permeation in bacterial sodium channels, Biochim. Biophys. Acta (BBA) - Biomembr. 1861, 495 - 503 (2019).

[24] Guardiani, C., Gibby, W. A. T., Barabash, M. L., Luchinsky, D. G. \& McClintock, P. V. E., Exploring the pore charge dependence of $\mathrm{K}^{+}$and $\mathrm{Cl}^{-}$permeation across a graphene monolayer: a molecular dynamics study, $R S C$ Adv. 9, 20402-20414 (2019).

[25] Gibby, W. A. T., Barabash, M. L., Guardiani, C., Luchinsky, D. G. \& McClintock, P. V. E., The role of noise in determining selective ionic conduction through nano-pores, in 2018 IEEE 13th Nanotechnology Materials and Devices Conference (NMDC) (IEEE, 2019).

[26] Zhan, H., Xiong, Z., Cheng, C., Liang, Q., Liu, J. Z. \& Li, D., Solvation-involved nanoionics: New opportunities from 2D nanomaterial laminar membranes, Adv. Mat. 32, 1904562 (2020).

[27] Smolyanitsky, A., Fang, A., Kazakov, A. F. \& Paulechka, E., Ion transport across solid-state ion channels perturbed by directed strain, Nanoscale 12, 10328-10334 (2020).

[28] Fedorenko, O. A., Kaufman, I. K., Gibby, W. A. T., Barabash, M. L., Luchinsky, D. G., Roberts, S. K. \& McClintock, P. V. E., Ionic Coulomb blockade and the determinants of selectivity in the $\mathrm{NaChBac}$ bacterial sodium channel, Biochim. Biophys. Acta (BBA) - Biomembranes 1862, 183301 (2020).

[29] Zwolak, M., Wilson, J. \& Ventra, M. D., Dehydration and ionic conductance quantization in nanopores, J. Phys.: Cond. Matt. 22, 454126 (2010).

[30] Gao, H., Shi, Q., Král, P. \& Lu, R., Water transport through ultrathin nanopores with highly polar rims, $J$. Phys. Chem. C 123, 27690-27696 (2019).

[31] Zhang, X., Wei, M., Xu, F. \& Wang, Y., Thicknessdependent ion rejection in nanopores, J. Membr. Sci. 601, 117899 (2020).

[32] Akaishi, A., Yonemaru, T. \& Nakamura, J., Formation of water layers on graphene surfaces, ACS Omega 2, 21842190 (2017).

[33] Mendonça, B. H., de Freitas, D. N., Köhler, M. H., Batista, R. J., Barbosa, M. C. \& de Oliveira, A. B., Diffusion behaviour of water confined in deformed carbon nanotubes, Physica A 517, 491 - 498 (2019).

[34] Chen, B., Jiang, H., Liu, X. \& Hu, X., Molecular insight into water desalination across multilayer graphene oxide membranes, ACS Applied Materials 85 Interfaces 9, 22826-22836 (2017), pMID: 28640581.

[35] Ballenegger, V. \& Hansen, J.-P., Dielectric permittivity profiles of confined polar fluids, J. Chem. Phys. 122, 114711 (2005).

[36] Bonthuis, D. J., Gekle, S. \& Netz, R. R., Profile of the static permittivity tensor of water at interfaces: 
Consequences for capacitance, hydration interaction and ion adsorption, Langmuir 28, 7679-7694 (2012), pMID: 22414296.

[37] Rigo, E., Dong, Z., Park, J. H., Kennedy, E., Hokmabadi, M., Almonte-Garcia, L., Ding, L., Aluru, N. \& Timp, G., Measurements of the size and correlations between ions using an electrolytic point contact, Nat. Comm. 10, 2382 (2019).

[38] Gibby, W. A. T., Barabash, M. L., Guardiani, C., Luchinsky, D. G., Fedorenko, O. A., Roberts, S. K. \& McClintock, P. V. E., Theory and experiments on multi-ion permeation and selectivity in the $\mathrm{NaChBac}$ ion channel, Fluct. Noise Lett. 18, 1940007 (2019).

[39] Barabash, M. L., Gibby, W. A. T., Guardiani, C., Luchinsky, D. G. \& McClintock, P. V. E., From the potential of the mean force to a quasiparticle's effective potential in narrow ion channels, Fluct. Noise Lett. 18, 1940006 (2019).

[40] Ruan, Y., Zhu, Y., Zhang, Y., Gao, Q., Lu, X. \& Lu, L., Molecular dynamics study of $\mathrm{Mg}^{2+} / \mathrm{Li}^{+}$separation via biomimetic graphene-based nanopores: The role of dehydration in second shell, Langmuir 32, 13778-13786 (2016), pMID: 27756127.

[41] Wang, Z.-Y. \& Wu, J., Ion association at discretelycharged dielectric interfaces: Giant charge inversion, $J$. Chem. Phys. 147, 024703 (2017).

[42] Amiri, H., Shepard, K. L., Nuckolls, C. \& Hernández Sánchez, R., Single-walled carbon nanotubes: Mimics of biological ion channels, Nano Lett. 17, 1204-1211 (2017).

[43] Novoselov, K. S., Geim, A. K., Morozov, S. V., Jiang, D., Zhang, Y., Dubonos, S. V., Grigorieva, I. V. \& Firsov, A. A., Electric field effect in atomically thin carbon films, Science 306, 666-669 (2004).

[44] Kirkwood, J. G. \& Boggs, E. M., The radial distribution function in liquids, J. Chem. Phys. 10, 394-402 (1942).

[45] Allen, M. P. \& Tildesley, D. J., Computer Simulation of Liquids (Clarendon Press, New York, NY, USA, 1987).

[46] McQuarrie, D. M., Statistical Mechanics (University Science Books, Sausalito, California, USA, 2000).

[47] Ben-Naim, A., Molecular Theory of Solutions (OUP Oxford, 2006).

[48] Egwolf, B., Luo, Y., Walters, D. E. \& Roux, B., Ion selectivity of $\alpha$-hemolysin with $\beta$-cyclodextrin adapter. ii. multi-ion effects studied with grand canonical Monte Carlo/Brownian dynamics simulations, J. Phys. Chem. B 114, 2901-2909 (2010), pMID: 20146515.

[49] Comer, J. \& Aksimentiev, A., Predicting the DNA sequence dependence of nanopore ion current using atomicresolution Brownian dynamics, J. Phys. Chem. C 116, 3376-3393 (2012).

[50] Hummer, G. \& Soumpasis, D. M., Computation of the water density distribution at the ice-water interface using the potentials-of-mean-force expansion, Phys. Rev. E 49, 591-596 (1994).

[51] Kirkwood, J. G., Statistical mechanics of fluid mixtures, J. Chem. Phys. 3, 300-313 (1935).

[52] Gray, C. G. \& Gubbins, K. E., Theory of Molecular Fluids (Oxford University Press, 1984).

[53] Hummer, G., García, A. \& Soumpasis, D., Hydration of nucleic acid fragments: comparison of theory and experiment for high-resolution crystal structures of rna, dna, and dna-drug complexes, Biophys. J. 68, 1639 - 1652 (1995).
[54] Soper, A. K., The radial distribution functions of water as derived from radiation total scattering experiments: Is there anything we can say for sure?, ISRN Phys. Chem. 2013, 67 (2013).

[55] Clayton, G. T. \& Heaton, L., Neutron diffraction study of krypton in the liquid state, Phys. Rev. 121, 649-653 (1961).

[56] Feig, M., Modeling Solvent Environments: Applications to Simulations of Biomolecules (WILEY-VCH Verlag GmbH \& Co. KGaA, Weinheim, 2010).

[57] Hirata, F., Molecular Theory of Solvation (Kluwer Academic Publishers, Dordrecht, 2004).

[58] Hansen, J. P. \& McDonald, I. R., Theory of Simple Liquids (Academic Press Inc. (London) Ltd., 1976).

[59] Beglov, D. \& Roux, B., An integral equation to describe the solvation of polar molecules in liquid water, J. Phys. Chem. B 101, 7821-7826 (1997).

[60] Jhon, Y. I., Kim, C., Byun, Y. T., Lee, J. H. \& Jhon, Y. M., Facile large-area fabrication of highly selective and permeable few-layered graphene: A molecular dynamics study, Carbon 155, 369 - 378 (2019).

[61] Rajan, G. A., Silmore, K. S., Swett, J., Robertson, A. W., Warner, J. H., Blankschtein, D. \& Strano, M. S., Addressing the isomer cataloguing problem for nanopores in two-dimensional materials, Nat. Mater. 18, 129-135 (2019).

[62] Beckstein, O., Tai, K. \& Sansom, M. S. P., Not ions alone: Barriers to ion permeation in nanopores and channels, J. Amer. Chem. Soc. 126, 14694-14695 (2004), pMID: 15535674.

[63] Barabash, M., Gibby, W., Smolyanitsky, A., Luchinsky, D., Luan, B. \& McClintock, P., Field-dependent dehydration and optimal ionic escape paths for $\mathrm{C}_{2} \mathrm{~N}$ membranes, J. Phys. Chem. C (2021), (in preparation).

[64] Gongadze, E. \& Iglič, A., Asymmetric size of ions and orientational ordering of water dipoles in electric double layer model - an analytical mean-field approach, Electrochimica Acta 178, 541-545 (2015).

[65] Fumagalli, L. et al., Anomalously low dielectric constant of confined water, Science 360, 1339-1342 (2018).

[66] Roux, B., Allen, T., Berneche, S. \& Im, W., Theoretical and computational models of biological ion channels, $Q$. Rev. Biophys 37, 15-103 (2004).

[67] Schoch, R. B., Han, J. \& Renaud, P., Transport phenomena in nanofluidics, Rev. Mod. Phys. 80, 839-883 (2008).

[68] Gillespie, D., Petsev, D. N. \& van Swol, F., Electric Double Layers with Surface Charge Regulation Using Density Functional Theory, Entropy 22, 132 (2020).

[69] Eisenberg, B., Hyon, Y. \& Liu, C., Energy variational analysis of ions in water and channels: Field theory for primitive models of complex ionic fluids, J. Chem. Phys. 133, 104104 (2010).

[70] Sato, T., Sasaki, T., Ohnuki, J., Umezawa, K. \& Takano, M., Hydrophobic surface enhances electrostatic interaction in water, Phys. Rev. Lett. 121, 206002 (2018).

[71] Varghese, S., Kannam, S. K., Hansen, J. S. \& P. Sathian, S., Effect of hydrogen bonds on the dielectric properties of interfacial water, Langmuir 35, 8159-8166 (2019), pMID: 31121091.

[72] Zhou, W., Wei, M., Zhang, X., Xu, F. \& Wang, Y., Fast desalination by multilayered covalent organic framework (cof) nanosheets, ACS Appl. Mater. Interfaces 11, 16847-16854 (2019).

[73] Sui, X., Yuan, Z., Yu, Y., Goh, K. \& Chen, Y., 2D mate- 
rial based advanced membranes for separations in organic solvents, Small 16, 2003400 (2020).

[74] Nocedal, J. \& Wright, S. J., Numerical optimization, Springer series in operations research (Springer, New York, 2006), 2nd ed edn., oCLC: ocm68629100.

[75] Dykman, M. I., Luchinsky, D. G., McClintock, P. V. E. \& Smelyanskiy, V. N., Corrals and critical behavior of the distribution of fluctuational paths, Phys. Rev. Lett. 77, 5229-5232 (1996).

[76] Luchinsky, D. G. \& McClintock, P. V. E., Irreversibility of classical fluctuations studied in analogue electrical circuits, Nature 389, 463-466 (1997).

[77] Guardiani, C., Barabash, M. L., Gibby, W. A. T., Luchinsky, D. G., Khovanov, I. A. \& McClintock, P. V. E., Prehistory probability distribution of ionic transitions through a graphene nanopore, in Proceedings of the 25th International Conference on Noise and Fluctuations (ICNF) (Eds. C. Enz \& F. Pascal) (EPFL, 2019).

[78] Yuan, Z., Liu, Y., Dai, M., Yi, X. \& Wang, C., Controlling DNA translocation through solid-state nanopores, Nanoscale Res. Lett 15 (2020).

[79] Materials Genome Initiative, https://www.nist.gov/ mgi, accessed: April 30, 2021.

[80] de Pablo, J. J. et al., New frontiers for the materials genome initiative, Npj Comput. Mater. 5 (2019).
[81] Marbach, S., Dean, D. S. \& Bocquet, L., Transport and dispersion across wiggling nanopores, Nat. Phys. 14, 1108-1113 (2018).

[82] Epsztein, R., DuChanois, R. M., Ritt, C. L., Noy, A. \& Elimelech, M., Towards single-species selectivity of membranes with subnanometre pores, Nat. Nanotechnol. (2020).

[83] Humphrey, W., Dalke, A. \& Schulten, K., VMD: Visual molecular dynamics, J. Mol. Graph. 14, 33 - 38 (1996).

[84] Phillips, J. C. et al., Scalable molecular dynamics with NAMD, J. Comput. Chem. 26, 1781-1802 (2005).

[85] Jorgensen, W. L., Chandrasekhar, J., Madura, J. D., Impey, R. W. \& Klein, M. L., Comparison of simple potential functions for simulating liquid water, J. Chem. Phys. 79, 926-935 (1983).

[86] Essmann, U., Perera, L., Berkowitz, M. L., Darden, T., Lee, H. \& Pedersen, L. G., A smooth particle mesh Ewald method, J. Chem. Phys. 103, 8577-8593 (1995).

[87] Matsunaga, Y., MDToolbox, https://github.com/ ymatsunaga/mdtoolbox (2018).

[88] MATLAB, https://www.mathworks.com, online.

[89] Luong, B., N-dimensional histogram, https: //uk.mathworks.com/matlabcentral/fileexchange/ 23897-n-dimensional-histogram (2011).

[90] Reeves, G., smooth2a, https://uk.mathworks.com/ matlabcentral/fileexchange/23287-smooth2a (2009). 